\title{
Evaluación de la eficacia del Programa de Pensamiento Prosocial (PPS)
}

\section{Evaluation of the effectiveness of the Reasoning and Rehabilitation Program (RRP)}

\author{
Miguel Contreras Orduño \\ Martha Frías Armenta \\ Universidad de Sonora
}

\begin{abstract}
Resumen
El objetivo del presente estudio fue conocer la eficacia del Programa de Pensamiento Prosocial (PPS) en una población de delincuentes jóvenes. La muestra consistió en 159 jóvenes internos en centros de internamiento en el estado de Sonora, 156 hombres y tres mujeres, de 14 a 22 años con promedio de edad de $16.7(D E=1.3)$. Se utilizaron las siguientes subescalas del test de Conners (Conners, 2000): inatención, impulsividad-hiperactividad, problemas de aprendizaje, agresión, problemas familiares, trastorno de inatención, trastorno de impulsividad e hiperactividad, trastorno disocial y trastorno negativista desafiante. A los jóvenes se les explicaron los objetivos de la evaluación, asimismo, se les pidió su consentimiento para participar y se les aseguró total confidencialidad de la información. Los encargados del levantamiento de datos fueron psicólogos entrenados, especialmente en el procedimiento de observación y aplicación de los instrumentos; el tiempo estimado de aplicación fue de 35 minutos. El instrumento se aplicó en las aulas de medios y salones de trabajo de los centros de tratamiento en internamiento. Se realizó una prueba $t$ para observar la diferencia entre el pre-test y el post-test. Los resultados mostraron diferencias significativas entre el pre-test y el post-test en todas las subescalas, lo cual sugiere que el PPS pudiera ser un tratamiento efectivo para los jóvenes en conflicto con la ley.

Palabras clave: violencia, delincuencia juvenil, programa de pensamiento prosocial, habilidades sociales.
\end{abstract}

Nota del autor

Miguel Contreras Orduño, Departamento de Psicología y Ciencias de la Comunicación, Universidad de Sonora (UNISON); Martha Frías Armenta, Departamento de Derecho, UNISON.

Esta investigación fue subvencionada por el Consejo Nacional de Ciencia y Tecnología de México (CONACYT) a través de la beca no. 7725.

La correspondencia en relación con este artículo debe dirigirse a Miguel Contreras Orduño, Departamento de Psicología y Ciencias de la Comunicación, UNISON, bulevar Luis Encinas y Rosales, s/n, colonia Centro, C. P. 83000, Hermosillo, Sonora, México.

Dirección electrónica: miguel.contrerasorduno@unison.mx 


\begin{abstract}
The objective of the present study was to evaluate the effectiveness of the Reasoning and Rehabilitation Program (RRP) in a Mexican population. The sample consisted on 159 young offenders institutionalized in treatment centers for adolescents in the state of sonora; 156 men and three women, from 14 to 22 years old $(M=16.7, S D=1.3)$. The following subscales of the test of Conners were used (Conners, 2000): inattention, impulsiveness-hiperactivity, learning problems, aggression, family problems, inattention disorder, impulsiveness and hyperactivity disorder, dissocial disorder and oppositional defiant disorder. It was explained the objectives of the evaluation and it was requested their consent to participate and it was assured total confidentiality of the information. The data was rising for psychologists trained especially in the observation procedure and application of the instruments; the time of application was between 35 minutes. The instruments were applied in the classrooms of the treatment centers. We used a t test to observe the difference between the pre-test and the post-test. Results showed significant differences between the pre-test and the post-test, in all the subscales, which demonstrated that the RRP is an effective program in the treatment of the participants in conflict with the law.
\end{abstract}

Keywords: violence, juvenile delinquency, reasoning and rehabilitation program, social abilities.

La violencia representa una amenaza que genera problemas para el sector salud, los sistemas de justicia, la sociedad, instituciones de tratamiento y la economía de las comunidades; en particular, repercuten en los niños que sufren abandono, negligencia, castigo físico fuerte y abuso sexual, e inclusive, los niños que son testigos de violencia en su casa o comunidad tienen un alto riesgo de cometer conductas antisociales en los años posteriores (World Health Organization, 2014). A nivel mundial, la mayor parte de las víctimas de homicidio son los jóvenes, representados por grupos de 15 a 29 años y de 30 a 44 años de edad; casi la mitad de todas las muertes sucedió a personas de entre $15 \mathrm{y}$ 29 años y poco menos de un tercio se encontraba entre los 30 y 44 años. En concreto, 36,000 niños menores de 15 años fueron víctimas de homicidio en todo el mundo durante 2012, lo que representó $8 \%$ del total de víctimas de ese delito (Oficina de las Naciones Unidas contra la Droga y Delito, 2013). En Estados Unidos, entre 1960 (400,000 casos) y 2011 (1236,200 casos) hubo un aumento de $205 \%$ en el número de casos de delincuentes juveniles con procesos judiciales, sin embargo, entre el pico más alto (1997, 1800,000 casos, aproximadamente) al 2011, hubo una disminución de 34\% (Hockenberry \& Puzzanchera, 2014). En México, el 23.1\% de los individuos que cometieron delitos tenían 25 años o menos (INEGI, 2014). Asimismo, los delincuentes juveniles atraviesan un periodo crítico para la persistencia o la resistencia delictiva, en el cual se torna fundamental realizar intervenciones especializadas que logren abordar las características y requerimientos 
propios de su situación (Richards, 2011); dicha etapa se caracteriza por presentar problemas de identidad, de relación con el cuerpo, de reconocimiento familiar y de establecimiento de las diferencias sexuales (Coslin, 2009). En las últimas décadas, el mito de que "nada funciona" en el área de prevención de la delincuencia y la rehabilitación ha sido reemplazado por una visión menos radical, según la cual algunos programas reducen la delincuencia (Howell, 2009; Van Voorhis, Braswell, \& Lester, 2004): en especial, el tratamiento cognitivo-conductual (Landenberger \& Lipsey, 2005) y los métodos conductual, cognitivo y conductual-cognitivo multimodal (McGuire, 2001). Consecuentemente, existe la necesidad de implementar programas que disminuyan la conducta antisocial en personas de riesgo desde la adolescencia; unos de los más efectivos han sido los socio-afectivos que favorecen el conocimiento y el manejo de las emociones (Silverio \& García, 2007). El PPS se ha implementado para atender esas necesidades socio-afectivas y de valores en los adolescentes; inició en 1966, después de una serie de investigaciones realizada como resultado del trabajo colaborativo del Departamento de Psicología de la Universidad de Waterloo y el Ministerio de Servicios Correccionales de Ontario, con el objetivo de brindar tratamiento a una institución de máxima seguridad para mujeres adolescentes delincuentes en una pequeña ciudad de Canadá (López, Garrido, \& Ross, 2001).
El PPS sido desarrollado y modificado en el Cognitive Centre of Canada (Cognitive Centre of Canada, 2015), el cual fue establecido en la Universidad de Ottawa en 1980, como un centro de investigación y desarrollo de programas de métodos efectivos para la prevención y el tratamiento de la conducta antisocial. Las actividades de este centro están dirigidas por un grupo de consultores expertos con vasta experiencia en distintos campos, incluyendo la psicología clínica, psicológica forense, neuropsicología, psicología cognitiva, educación, criminología, justicia criminal y juvenil, evaluación de programas y entrenamiento profesional. Más tarde, en 1986 desarrolló y acreditó internacionalmente el programa de rehabilitación y razonamiento, un manual del programa en tres volúmenes: Reasoning and rehabilitation: an handbook for teaching cognitive skills (Ross, Fabiano, \& Ross, 1986), constituido por 35-38 sesiones, con una duración de dos horas. Su enfoque se centra en la enseñanza de habilidades cognitivas, sociales y valores que son antagónicos a la conducta antisocial y esenciales para la realización de competencias prosociales. Asimismo, dicho manual toma como base el libro: Time to Think: A cognitive model of offender rehabilitation and delinquency prevention de Ross y Fabiano (1985).

El programa se ha impartido en instituciones para jóvenes que cometen conductas antisociales como prisiones y lugares de media estancia, hospitales psiquiátricos forenses, hospitales 
para desórdenes mentales, durante libertad condicional, agencias de servicio social comunitario, en escuelas especiales y en escuelas de la comunidad (Cognitive Centre of Canada, 2015), así como en niños y adolescentes en riesgo. Actualmente es el programa central del Servicio Correccional de Canadá, y funciona en 47 prisiones y residencias comunitarias para los delincuentes más peligrosos de toda la nación (López et al., 2001).

El PPS ha sido aplicado en 22 países (Cognitive Centre of Canada, 2015), incluyendo Australia, Canadá, Islas Canarias, Dinamarca, Inglaterra (Liverpool, Londres y Manchester), Estonia, Finlandia, Alemania, Holanda, Irán, Hong Kong, Jersey, Letonia, Líbano, Nueva Zelanda, Noruega, Estonia, Escocia, España (Barcelona, Valencia), Suecia, Suiza, Emiratos Árabes Unidos, Gales y muchos estados de Estados Unidos de Norteamérica (Alaska, California, Connecticutt, Massachussets, Michigan, Minnesota, New Jersey, New York, Nuevo México, Ohio, Oregon, Texas, Utah, Vermonty Washington), además, ha sido aplicado a delincuentes adolescentes institucionalizados y analfabetas de barrios marginales de Caracas.

En España (Alba et al., 2005), adaptaron una versión corta para jóvenes del PPS, con base en los libros de Robert Ross: Time to think again (Ross \& Hilborn, 2003a) y Rasoning and rehabilitation 2 (Ross \& Hilborn, 2003b).

Esta versión consta de las siguientes sesiones (Alba et al., 2005):

- Sesión 0: Sesión introductoria.

- Sesión 1: Habilidades del pensamiento, sentimientos y conducta.
- Sesión 2: Control de los pensamientos, emociones y conductas (parte 1).

- Sesión 3: Control de los pensamientos, emociones y conductas (parte 2 ).

- Sesión 4: Búsqueda de información.

- Sesión 5: Reconocer pensamientos y emociones.

- Sesión 6: Búsqueda de alternativas.

- Sesión 7: Detección de los errores de pensamiento propios y de los demás.

- Sesión 8: Pensar en las consecuencias de los actos propios y de los demás.

- Sesión 9: Elegir las alternativas válidas.

- Sesión 10: Aplicación y puesta en marcha de la alternativa elegida.

- Sesión 11: Desarrollo y cambio de valores.

- Sesión 12: Manejo de conflictos.

Dicho contenido es impartido en dos sesiones semanales (se concluye el programa en mes y medio aproximadamente); requiere de pocos recursos: uno o dos monitores, computadora, cañón, cartulina, pizarrón y papelería en general. El programa no está diseñado para tratar directamente los problemas personales que los delincuentes puedan experimentar sino para equiparlos con habilidades y actitudes que les ayudarán a evitar problemas. Se asume que la mejor manera de rehabilitar es de naturaleza educativa, que entrena directa y sistemáticamente en técnicas, actitudes $\mathrm{y}$ valores para capacitar al delincuente de una manera eficaz, con habilidades prosociales y valores específicos, cuyo fin es habilitarlos para escoger un estilo de vida adaptado frente al comportamiento antisocial. 
Esta versión fue la que se utilizó en el Instituto de Tratamiento y de Aplicación de Medidas para Adolescentes (ITAMA), en el estado de Sonora, México. Por lo tanto, el objetivo de esta investigación fue evaluar la efectividad del PPS en una población de jóvenes delincuentes, con el fin de poder contar con un programa preventivo y de tratamiento para jóvenes en riesgo (en distintos niveles) de cometer conductas antisociales y delictivas. La hipótesis de investigación fue la siguiente: El programa de pensamiento prosocial tiene una relación negativa con los problemas de atención, impulsividad-hiperactividad, aprendizaje, agresividad, y familiares, además de los trastornos de atención, impulsividad e hiperactividad, disocial y negativista desafiante de los jóvenes en conflicto con la ley.

\section{Método}

\section{Participantes}

Los participantes del presente estudio fueron 159 jóvenes, internos en los centros de tratamiento en internamiento sonorenses, de éstos, 156 eran hombres y tres eran mujeres; la población se ubicaba entre los 14 y 22 años con promedio de edad de 16.7 y una desviación estándar de 1.3. El diseño de la investigación fue cuasi-experimental pre-test y post-test. El tipo de muestra fue no probabilístico por conveniencia.

En la tabla 1 podemos observar que el promedio de años de escolaridad es de $7.6 \mathrm{y}$ la desviación estándar es de 2.3; la mínima de escolaridad fue de ningún grado cursado y la máxima de tres años de preparatoria.

Tabla 1

Escolaridad de los adolescentes

\begin{tabular}{lcccc}
\hline \multicolumn{1}{c}{ Escolaridad } & Frecuencia & Porcentaje & Porcentaje válido & $\begin{array}{c}\text { Porcentaje } \\
\text { acumulado }\end{array}$ \\
\hline Sin estudios & 5 & 3.1 & 3.1 & 3.1 \\
2do grado & 2 & 1.3 & 1.3 & 4.4 \\
3er grado & 2 & 1.3 & 1.3 & 5.7 \\
4to grado & 8 & 5.0 & 5.0 & 10.7 \\
5to grado & 4 & 2.5 & 2.5 & 13.2 \\
6to grado & 24 & 15.1 & 15.1 & 28.3 \\
1ro de secundaria & 15 & 9.4 & 9.4 & 37.7 \\
2do de secundaria & 16 & 10.1 & 10.1 & 47.8 \\
3ro de secundaria & 64 & 40.3 & 40.3 & 88.1 \\
1 año de preparatoria & 13 & 8.2 & 8.2 & 96.2 \\
2 año de preparatoria & 5 & 3.1 & 3.1 & 99.4 \\
3 año de preparatoria & 1 & 0.6 & 0.6 & 100 \\
\hline Total & 159 & 100 & 100 & \\
\hline
\end{tabular}


Los centros de internamiento se encuentran en Hermosillo, con la excepción del centro del sur de Sonora, que se encuentra en Cócorit, municipio de Cajeme. En la tabla 2 podemos observar que más de $46 \%$ de los adolescentes que participaron en el programa estaban internados en el centro intermedio.
El 74.2\% reveló consumir algún tipo de droga y 24.5 lo negó. En la tabla 3 se pueden apreciar los niveles de consumo de droga de los participantes.

\section{Tabla 2}

Centros de internamiento de los adolescentes

\begin{tabular}{lcccc}
\hline \multicolumn{1}{c}{ Centro } & Frecuencia & Porcentaje & $\begin{array}{c}\text { Porcentaje } \\
\text { válido }\end{array}$ & $\begin{array}{c}\text { Porcentaje } \\
\text { acumulado }\end{array}$ \\
\hline Sur de Sonora Cócorit & 55 & 34.6 & 34.6 & 34.6 \\
Granja Marco Antonio & 27 & 17.0 & 17.0 & 51.6 \\
Salazar Siqueiros & 74 & 46.5 & 46.5 & 98.1 \\
Intermedio & 3 & 1.9 & 1.9 & 100 \\
Granja San Antonio & 159 & 100 & 100 & \\
Total & & & & \\
\hline
\end{tabular}

Tabla 3

Consumo de drogas

\begin{tabular}{lcccc}
\hline \multicolumn{1}{c}{ Droga } & Sí consume & Porcentaje & $\begin{array}{c}\text { No consume } \\
\text { (2 datos perdidos) }\end{array}$ & Porcentaje \\
\hline Marihuana & 104 & 65.4 & 53 & 33.3 \\
Cocaína & 12 & 7.5 & 145 & 91.2 \\
Cristal & 49 & 30.8 & 108 & 67.9 \\
Alcohol & 41 & 25.8 & 116 & 73.0 \\
Tabaco & 69 & 43.4 & 88 & 55.3 \\
Pastillas & 39 & 24.5 & 118 & 74.2 \\
Heroína & 5 & 3.1 & 152 & 95.6 \\
Spray & 6 & 3.8 & 151 & 95.0 \\
Resistol & 12 & 7.5 & 145 & 91.2 \\
\hline
\end{tabular}

Por otro lado, la tabla 4 resalta que el delito de mayor frecuencia fue el robo agravado con $48.4 \%$, mismo que puede ser caracterizado por haberse efectuado con violencia, con arma de fuego o blanca, en casas o comercios, cometido de noche, por una, dos o más personas. Asimis- 
mo, el robo en cualquiera de sus modalidades se presenta con mayor frecuencia; en este caso, aunque menos grave, se observa alta frecuencia del robo simple y el robo a casa habitación, sin embargo, los delitos contra la salud presentan el segundo lugar de frecuencia, 7.5\%. Por otro lado, cabe resaltar que más de $12 \%$ de los ado- lescentes cometió homicidio (simple, simple doloso, calificado y en grado de tentativa). Finalmente, de acuerdo con la clasificación de Clinard y Quinney (1973), el porcentaje de crímenes violentos (asesinato, violación y robo agravado) de los participantes ascendería a más del $67 \%$.

Tabla 4

Delitos de los adolescentes

\begin{tabular}{lcccc}
\hline \multicolumn{1}{c}{ Delito } & Frecuencia & Porcentaje & $\begin{array}{c}\text { Porcentaje } \\
\text { valido }\end{array}$ & $\begin{array}{c}\text { Porcentaje } \\
\text { acumulado }\end{array}$ \\
\hline Robo simple & 6 & 3.8 & 3.8 & 3.8 \\
Robo en casa habitación & 11 & 6.9 & 6.9 & 10.7 \\
Robo agravado & 77 & 48.4 & 48.4 & 59.1 \\
Robo de vehículo & 7 & 4.4 & 4.4 & 63.5 \\
Lesiones simples & 2 & 1.3 & 1.3 & 64.8 \\
Lesiones que tardan más de quince días & 1 & .6 & .6 & 65.4 \\
para sanar y ponen en riesgo la vida & & & 7.5 & 73.0 \\
Delitos contra la salud & 12 & 1.9 & 1.9 & 74.8 \\
Abusos deshonestos & 3 & 3.8 & 3.8 & 78.6 \\
Violación & 6 & 3.1 & 3.1 & 81.8 \\
Violación equiparada & 5 & 1.9 & 1.9 & 83.6 \\
Secuestro & 3 & 4.4 & 4.4 & 88.1 \\
Homicidio simple & 7 & 1.3 & 1.3 & 89.3 \\
Homicidio simple doloso & 2 & 3.8 & 3.8 & 93.1 \\
Homicidio calificado & 6 & 3.1 & 3.1 & 96.2 \\
Homicidio en grado de tentativa & 5 & .6 & .6 & 96.9 \\
Violación a la ley federal de armas de fuego & 1 & .6 & .6 & 97.5 \\
y explosivos & 1 & 2.5 & 2.5 & 100 \\
Violación a la ley general de población & 4 & 100 & 100 & \\
Portación de arma blanca & 159 & & & \\
\hline Total & & & & \\
\hline
\end{tabular}


El 78.0\% de los adolescentes manifestó ser primo-infractor. Por último, en la tabla 5 se puede observar que $12.7 \%$ ha estado tres veces internado por cometer algún delito.

Tabla 5

Reincidencia de los participantes

\begin{tabular}{ccccc}
\hline Número de reincidencia & Frecuencia & Porcentaje & Porcentaje válido & $\begin{array}{c}\text { Porcentaje } \\
\text { acumulado }\end{array}$ \\
\hline 0 & 124 & 78 & 79 & 79 \\
1 & 2 & 1.3 & 1.3 & 80.3 \\
2 & 20 & 12.6 & 12.7 & 93.0 \\
3 & 8 & 5 & 5.1 & 98.1 \\
4 & 1 & .6 & .6 & 98.7 \\
6 & 1 & .6 & .6 & 99.4 \\
7 & 1 & .6 & .6 & 100 \\
\hline Total & 157 & 98.7 & 100 & \\
\hline
\end{tabular}

\section{Instrumentos}

Para el levantamiento de datos se utilizó una plantilla con variables sociodemográficas y las siguientes subescalas del test de Conners (Conners, 2000): inatención (11 reactivos), impulsividad-hiperactividad (14 reactivos), problemas de aprendizaje (8 reactivos), agresión (15 reactivos), problemas familiares (8 reactivos), trastorno de inatención (11 reactivos), trastorno de impulsividad e hiperactividad (11 reactivos), trastorno disocial (14 reactivos) y trastorno negativista desafiante ( 8 reactivos). Las escalas de Conners (2000) se fundamentan en los criterios del manual diagnóstico y estadístico de los trastornos mentales. Estos criterios son las más utilizados para diagnosticar los trastornos disociales (De la Peña, 2003). Gallant, Conners, Rzepa, Pitkanen, Marocco y Sitarenios (2007, citados por Conners, 2000) obtuvieron una consistencia interna con el alfa de Cronbach: inatención .92, impulsividad-hiperactividad .89 , problemas de aprendizaje .84, agresión .85, problemas familiares .88 , trastorno de inatención .89 , trastorno de impulsividad e hiperactividad .85 , trastorno disocial $.81 \mathrm{y}$ trastorno negativista desafiante .83. Para observar la estabilidad de la escala por medio de test-retest se obtuvieron las siguientes correlaciones $(r)$ : inatención .90, impulsividad-hiperactividad .80, problemas de aprendizaje .74, agresión .95, problemas familiares .67, trastorno de inatención .78, trastorno de impulsividad e hiperactividad .75, trastorno disocial .91 y trastorno negativista desafiante .83 (Gallant, 2008, citado por Conners, 2000). En México, Contreras (2013) obtuvo las siguientes alfas de Cronbach: 
inatención .87, impulsividad-hiperactividad .74, problemas de aprendizaje .73, agresión .89, problemas familiares .63, trastorno de inatención .81 , trastorno de impulsividad e hiperactividad .81 , trastorno disocial .88 y trastorno negativista desafiante .73 .

\section{Procedimiento}

La recogida de datos se efectuó de manera grupal (de ocho a diez participantes, aproximadamente). Al acudir a los centros de tratamiento, fue solicitado el consentimiento de los jóvenes para participar, no sin antes haberles explicado el objetivo del estudio e informado que éste se llevaría a cabo en dos etapas, antes de empezar el PPS y al concluir el mismo. El tiempo de aplicación fue de aproximadamente 35 minutos por cada grupo. Los instrumentos fueron administrados por psicólogas entrenadas en técnicas de observación y entrevista en las aulas donde los adolescentes recibían las clases. Los instru- mentos fueron aplicados antes de empezar el programa y después de haberlo concluido. El tiempo entre pre-test y el post-test fue de aproximadamente dos meses.

\section{Análisis de datos}

Primeramente, se llevaron a cabo estadísticas univariadas, medias y desviaciones estándar para variables continuas, frecuencias para variables categóricas y alfas para todas las escalas. Una vez hecho esto, se elaboraron índices para cada una de las escalas y se midieron las diferencias de medias entre el pre-test y el post-test en cada una de ellas. Para este fin, se empleó el paquete estadístico SPSS, versión 21.

\section{Resultados}

En la tabla 6 se puede observar el alfa de todas las escalas, mismo que obtuvieron un alfa mayor a 0.67 .

Tabla 6

Alfa, medias y desviaciones estándar de la escala de inatención pre-test

\begin{tabular}{lccc}
\hline \multirow{2}{*}{ Escala y variable } & $N$ & \multicolumn{2}{c}{ Alfa } \\
\cline { 3 - 4 } & & Pre-test & Post-test \\
\hline Inatención & 159 & 0.85 & 0.89 \\
Impulsividad-hiperactividad & 159 & 0.76 & 0.82 \\
Problemas de aprendizaje pre-test & 159 & 0.79 & 0.83 \\
Agresión & 159 & 0.91 & 0.92 \\
Problemas familiares & 159 & 0.67 & 0.70 \\
Trastornos de atención, tipo inatento & 159 & 0.79 & 0.85 \\
Trastornos de atención, tipo impulsivo-hiper- & 159 & 0.79 & 0.83 \\
activo & & & \\
Trastorno disocial & 159 & 0.91 & 0.91 \\
Trastorno negativista desafiante & 159 & 0.70 & 0.80 \\
\hline
\end{tabular}


En el pre-test de la escala de inatención los reactivos que puntuaron más alto fueron "Me distraigo cuando pasa algo alrededor de mí" $($ media $=1.45)$ y "Dificultad para concentrarme" $($ media $=1.21)$. Los resultados de la escala pretest de impulsividad-hiperactividad mostraron que los adolescentes tienen mucha energía (media de 1.43), y que si se emocionan se ponen muy activos (media de 1.99). En relación con la escala de impulsividad-hiperactividad, los puntajes más altos presentados en el pre-test disminuyeron, a excepción del reactivo "Hago las cosas antes de pensar" y "Me emociono y me pongo muy activo"; esto puede deberse al tratamiento, ya que a los adolescentes se les entrenó en el control de sus emociones, pensamientos y conductas (sesión 2 y 3), habilidad necesaria para posteriormente poder solucionar problemas. Esto significa que cuando enfrenten problemas, el efecto fisiológico y emocional será menos intenso y podrán usar dichas habilidades de autocontrol efectivamente.

El PPS no busca directamente solucionar los problemas de aprendizaje que presentan los adolescentes en conflicto con la ley, sin embargo, entre el pre-test y el post-test se observó una disminución importante en todos los reactivos, con excepción del reactivo relacionado con la dificultad en las matemáticas.

El pre-test de la escala de agresión arrojó puntajes más altos en las conductas que se relacionan directamente con la comisión de conductas delictivas: "Me meto en problemas con la policía" (media de 1.43), "Me he escapado de casa" (media de 1.47), "Salgo en la noche cuando debo estar en casa" (media de 1.87) y "Cuando me enojo me desquito" (1.06). Asimismo, estos reactivos tuvieron una desviación estándar mayor que la de los otros reactivos (excepción de "robo objetos" y "utilizo arma"). En el post-test tales conductas antisociales disminuyeron.

El puntaje más alto obtenido por los adolescentes en el pre-test de la escala de problemas familiares se presentó en dos reactivos específicos: "Mis padres solo se fijan en mi mala conducta", con una media de $1.09 \mathrm{y}$ una desviación estándar de 1.11 y "Mis padres esperan demasiado de mí", con una media de 1.68 y una desviación estándar de 1.02.

En relación con la escala de trastornos de la atención tipo inatento, podemos destacar que en el pre-test ocho reactivos obtuvieron un puntaje por arriba de uno y en el post-test sólo cinco ("Dificultad para poner atención a los detalles", "Se me olvidan cosas", "Cometo errores", "No hago lo que me hace pensar mucho" y "Me distraigo cuando pasa algo alrededor de mí”).

En la escala de los trastornos de la atención tipo impulsivo-hiperactivo, los reactivos que puntuaron más alto fueron "Soy inquieto" (media de 1.47), "Hablo demasiado" (media de 1.15), "Me gusta estar moviéndome" (media de 1.65) y "Es difícil mantenerme quieto" (media de 1.31), los cuales disminuyeron en el post-test, con excepción del reactivo "Hablo demasiado".

Los resultados de las escalas del trastorno disocial mostraron una disminución en todos los reactivos del pre-test al post-test. La escala del trastorno negativista desafiante arrojó algunos resultados interesantes. En primer lugar, 
los reactivos que se asocian más con conductas antisociales: "Discuto con los adultos", "Me descontrolo, pierdo la paciencia, me enojo", "Me enojo y me desquito" y "La gente me hace enojar", presentaron puntajes más altos en comparación con los demás reactivos. En segundo lugar, los reactivos que se asocian más con conductas antisociales, mencionados anteriormente, disminuyeron después del tratamiento.

Finalmente, en la tabla 7 se observan diferencias significativas entre el pre-test y el post-test de todas las subescalas del test de Conners.

\section{Tabla 7}

Diferencias entre el pre-test y post-test

\begin{tabular}{|c|c|c|c|c|c|}
\hline Escala & $N$ & Media & $D E$ & $t(g l)$ & Nivel de significancia \\
\hline Inatención pre & 159 & 1.09 & 0.59 & $2.58(158)$ & 0.011 \\
\hline Inatención post & 159 & .99 & 0.62 & & \\
\hline Impulsividad-hiperactividad pre & 159 & 1.20 & 0.48 & $2.95(158)$ & 0.00 \\
\hline Impulsividad-hiperactividad post & 159 & 1.10 & 0.51 & & \\
\hline Problemas de aprendizaje pre & 159 & 1.22 & 0.62 & $2.27(158)$ & 0.02 \\
\hline Problemas de aprendizaje post & 159 & 1.14 & 0.66 & & \\
\hline Agresión pre & 159 & 1.02 & 0.66 & $4.42(158)$ & 0 \\
\hline Agresión post & 159 & 0.82 & 0.65 & & \\
\hline Problemas familiares pre & 159 & 0.83 & 0.49 & $2.53(158)$ & 0.01 \\
\hline Problemas familiares post & 159 & 0.74 & 0.48 & & \\
\hline Ts de atención tipo inatento pre & 159 & 1.14 & 0.51 & $2.05(158)$ & 0.04 \\
\hline Ts de atención tipo inatento post & 159 & 1.06 & 0.55 & & \\
\hline $\begin{array}{l}\text { Ts de atención tipo impulsivo- } \\
\text { hiperactivo pre }\end{array}$ & 159 & 1.08 & 0.55 & $2.67(158)$ & 0.001 \\
\hline $\begin{array}{l}\text { Ts de atención tipo impulsivo- } \\
\text { hiperactivo post }\end{array}$ & 159 & 0.99 & 0.56 & & \\
\hline Ts disocial pre & 159 & 0.99 & 0.68 & $4.23(158)$ & 0 \\
\hline Ts disocial post & 159 & 0.79 & 0.65 & & \\
\hline Ts negativista desafiante pre & 159 & 1.09 & 0.52 & $2.54(158)$ & 0.01 \\
\hline Ts negativista desafiante post & 159 & 0.99 & 0.58 & & \\
\hline
\end{tabular}




\section{Discusión}

De acuerdo con los resultados obtenidos, se encontraron diferencias significativas en todas las subescalas del Conners, lo que nos pudiera indicar que el PPS es efectivo al disminuir los problemas de atención, impulsividad-hiperactividad, aprendizaje, agresividad, y familiares, además de los trastornos de atención, impulsividad e hiperactividad, disocial, y negativista desafiante de los jóvenes en conflicto con la Ley. Esto nos pudiera indicar que el programa sería efectivo en reducir los factores de riesgo correlacionados con la delincuencia juvenil, así como representar un tratamiento efectivo para prevenir y disminuir la delincuencia juvenil. En otras investigaciones, se ha demostrado que el PPS es un programa efectivo para reducir la delincuencia (Antonowicz, 2005; Cognitive Centre of Canada, 2015; Contreras, 2013; Frías, Hernández, \& Contreras, 2012; Tong \& Farrington, 2006, 2008; Wettermann, Schlafke, \& Fegert, 2012).

En esta investigación se encontró que el programa disminuyó los problemas de inatención, impulsividad-hiperactividad, los problemas de aprendizaje, la agresión, mejoró las relaciones familiares, así como disminuyó los trastornos de atención (trastorno tipo inatento e impulsivohiperactivo), disocial y negativista desafiante. Se puede señalar que el PPS es un programa que impacta principalmente en los factores de riesgo y su implementación es relevante para tratar la delincuencia juvenil, en especial, los factores señalados por Schreck, Miller y Gibson (2009): individuales, escolares y comunitarios. Por lo tanto, de los resultados obtenidos podemos concluir que las mejoras encontradas después de la aplicación del PPS podrían impactar de la siguiente manera: en lo que respecta a los factores individuales que influyen en la victimización, exposición, y lo atractivo que pudiera ser el crimen, las habilidades de pensamiento crítico les brindarían herramientas para enfrentar este tipo de situaciones. Con base en esta investigación, se considera que la disminución de la agresión $\mathrm{y}$ de las conductas antisociales favorecerían las actitudes que tienen los adolescentes hacia las conductas y los pares prosociales. Las habilidades sociales les permitirían, por su parte, mejorar las relaciones familiares y generar vínculos prosociales. Con respecto a los factores escolares que influyen en la victimización, es imposible controlar la desorganización social de sus escuelas con este programa, sin embargo, la disminución en general de los trastornos de atención, impulsividad e hiperactividad y los problemas escolares producto de haber cursado el PPS, les permitirían mejorar sus calificaciones y establecer también vínculos prosociales en la escuela. Esto es importante porque el poco éxito académico, la deserción escolar y el escaso compromiso con la educación conforman una de las características de los delincuentes juveniles (Henggeler, Schoenwald, Borduin, Rowland, \& Cunningham, 1998). Desde el punto de vista de los factores comunitarios, el PPS no impactaría directamente en las comunidades desorganizadas, sin embargo, la mejora en las 
relaciones familiares que lograron los participantes podría aumentar la supervisión parental, optimizar la disciplina, promover un ambiente de calidez en casa, para así disminuir la movilidad de los adolescentes. Además, éstos estarían en condiciones de promover la cohesión social en su comunidad.

Con respecto a las escalas de trastornos de la atención, se observó que los participantes presentan algunas dificultades. Por lo tanto, podemos concluir que tales dificultades podrían afectar todas las esferas donde se desenvuelven los adolescentes, y generarles muchos problemas para realizar sus actividades diarias e, inclusive, evadirlas. En relación con las variables exógenas (reincidencia, delitos, consumo de drogas) que afectan la eficacia del programa, a pesar de que $74 \%$ reveló consumir una droga, más de 28\% reportó ser policonsumidor, y 67\% cometió crímenes violentos, el programa demostró su efectividad, lo cual significa que si el PPS es utilizado también en adolescentes en riesgo que no presentan un historial delictivo, podría beneficiarlos y así prevenir de manera efectiva la delincuencia juvenil. Es decir, podría ser utilizado (como ya lo ha sido en otros países) con adolescentes en escuelas, comunidades e instituciones que tengan factores de riesgo para cometer conductas delictivas.

El programa presenta otro beneficio, al no requerir de profesionales de la salud mental altamente entrenados para impartirlo, sino que se trata de un programa que ha sido desarrollado para poder ser aplicado por personal competente, cualquiera que sea su formación académica (López et al., 2001); sin embargo, Contreras (2013) señala que es muy importante la motivación y los conocimientos del programa por parte de quien lo imparte. Por lo tanto, para combatir este tipo de condiciones (y otras) es necesaria la supervisión y evaluación del mismo. Si se siguen estas recomendaciones se mejorarían sus efectos y aumentaría la motivación de los adolescentes que participen, al tiempo que disminuirían las conductas antisociales de los mismos.

\section{Referencias}

Agnew, R. (2009). Foundation for a general strain theory of crime and delinquency. En R. Sacarpitti, L. Nielsen \& M. Miller (Eds.), Crime and criminals, contemporary and classic readings in criminology. New York: Oxford University Press.

Alba, J. L., Garrido, G. V., López, M. J., Burgues, M., Alcázar, M., López, J...Chordi, F. (2005). El Programa del Pensamiento Prosocial (versión corta para jóvenes). En G. V. Garrido \& M. J. López (Eds.), Manual de intervención educativa en readaptación social. Valencia: Tirant lo Blanch.

Akers, R. L (2009). A social learning theory of crime. En R. Sacarpitti, L. Nielsen \& M. Miller (Eds.), Crime and criminals, contemporary and classic readings in criminology. New York: Oxford University Press. 
Antonowicz, D. H. (2005). The reasoning and Rehabilitation Program: Outcome Evaluations with Offenders. En M. McMurran \& J. McGuirre (Eds.), Social Problem Solving and Offending: Evidence, Evaluation and Evolution (pp. 163-182). Chichester, UK: John Wiley \& Sons LTD. doi: 10.1002/9780470713488.ch9

Beaver, K. M., \& Walsh, A. (2011). Biosocial Criminology. En M. Beaver \& A. Walsh (Eds.), The ashgate research companion to biosocial theories of crime. Great Britain: Ashgate.

Cognitive Centre of Canada. (2015). Recuperado de http://www.cognitivecentre.ca/

Conners, K. (2000). Evaluation ADHD (3rd ed.). United States: MHS.

Contreras, O. M. (2013). Evaluación y adaptación del Programa de Pensamiento Prosocial (PPS) en una población de delincuentes juveniles en el Estado de Sonora, México (Tesis inédita de doctorado). Universidad de Sonora, México.

Coslin, P. G. (2009). Adolescencia y Violencia.

En M. Frías \& V. Corral (Eds), Niñez, adolescencia y problemas sociales. México: Unison y PyV.

Clinard, M. B., \& Quinney, R. (1973). Criminal behavior systems: A typology. New York: Holt, Rinehart and Winston.

De la Peña, O. F. (2003). Tratamiento multisistémico en adolescentes con trastornos disociales. Salud Pública de México, 45(1), 124131.
Garrido, G. V., \& López, M. J. (Eds.). (2005). Manual de intervención educativa en readaptación social. Valencia: Tirant lo Blanch.

Frías, A. M., Hernández, C. T., \& Contreras O. M. (2012). Sistema de justicia juvenil en México: Funcionamiento, intervención y evaluación. EduPsykhé, 11, 317-341.

Henggeler, S. W., Schoenwald, S. K., Borduin, Ch. M., Rowland, M. D., \& Cunningham, P. B. (1998). Multisystemic treatment of antisocial behavior in children and adolescent. New York: The Guilford Press.

Hirschi, T. (2009). A control theory of delinquency. En R. Sacarpitti, L. Nielsen \& M. Miller (Eds.), Crime and criminals, contemporary and classic readings in criminology. New York: Oxford University Press.

Hockenberry, S., \& Puzzanchera, Ch. (2014). Juvenil Court Statitistic 2011. Report. Pittsburg, PA: National Center for Juvenile Justice.

Howell, J. C. (2009). Preventing and reducing juvenile delinquency. Thousand Oaks, CA: Sage.

Huff, C. R. (2009). Historical explanations of crime: From demons to politics. En R. Sacarpitti, L. Nielsen \& M. Miller (Eds.), Crime and criminals, contemporary and classic readings in criminology. New York: Oxford University Press.

Instituto de Justicia Procesal Penal. (2013). Informe sobre justicia para adolescentes en conflicto con la ley penal en México. Estado actual después de las reformas constitucio- 
nales de 2005, 2008 y 2011. México: Fondo Canadá e Instituto de Justicia Procesal Penal. Instituto Nacional de Estadística y Geografía [INEGI]. (2014). Encuesta Nacional de Victimización y Percepción sobre seguridad pública (ENVIPE). Recuperado de www.inegi.org.mx/est/contenidos/proyectos/encuestas/hogares/regulares/envipe/default.aspx

Landenberger, N., \& Lipsey, M. (2005). The positive effects of cognitive-behavioural programs for offenders: A meta-analysis of factors associated with effective treatment. Journal of Experimental Criminology, 1(4), 451-476.

Lara-Muñoz, M., De la Peña, O. F., Castro, A., \& Puente, A. (1998). Consistencia y validez de las subescalas del cuestionario de Conners para la evaluación de psicopatología en niños-versión larga para padres. Bol Med Hosp Infant Mex, 55(12), 712-720.

López, M. J., Garrido, V., \& Ross, R. R. (2001). En busca del programa. En M. J. López, V. Garrido \& R. R. Ross (Eds.), El Programa del pensamiento prosocial: Avances recientes. Valencia: Tirant lo Blanch.

McGuire, J. (2001). Enfoques psicosociales para comprender y reducir la violencia en los jóvenes. En V. Varma (Ed.), La violencia en niños y adolescentes. México: Trillas.

Oficina de las Naciones Unidas contra la Droga y Delito. (2013). Estudio mundial sobre el homicidio. Resumen Ejecutivo. Recuperado de https://www.unodc.org/documents/gsh/ pdfs/GLOBAL_HOMICIDE_Report_ExSum_spanish.pdf

Popper, Ch., \& West, S. A. (2000). Trastornos de inicio en la infancia, la niñez o la adolescencia. En R. Hales, S. Yudofsky \& J. Talbott (Eds.), Tratado de psiquiatría (tomo I, 3rd ed.). España: Masson.

Reyes, E. A. (1999). Criminología. Colombia: Temis.

Richards, K. (2011). Technical and background paper: Measuring juvenile recidivism in Australia. Australia: AIC Reports. Australian Government. Australian Institute of Criminology.

Ross, R. R., \& Fabiano, E. (1985). Time to Think: A cognitive model of offender rehabilitation and delinquency prevention. Canadá: Air and Publications.

Ross, R., Fabiano, E., \& Ross, R. D. (1986). Reasoning and Rehabilitation: a handbook for teaching cognitive skills. Canadá: University of Otawa.

Ross, R. R., \& Hilborn, R. D. (2003a). Time to think again. Canadá: Air Training and Publications.

Ross, R. R., \& Hilborn, R. D. (2003b). Reasoning and Rehabilitation 2 Short Version. Canadá: Air Training and Publications.

Rowe, D. (2009). Does the body tell? Biological characteristics and criminal disposition. En R. Sacarpitti, L. Nielsen \& M. Miller (Eds), 
Crime and criminals, contemporary and classic readings in criminology. New York: Oxford University Press.

Rudo-Hutt, A., Gao, Y., Glenn, A., Peskin, M., Yanag, Y., \& Raine, A. (2011). Biosocial interactions and correlates of crime. En M. Beaver \& A. Walsh (Eds), The ashgate research companion to biosocial theories of crime. Great Britain: Ashgate.

Schreck, C. J., Miller, J. M., \& Gibson, C. L. (2009). Trouble in the school Yard: A study of the risk factors of victimization on the school. En R. Sacarpitti, L. Nielsen \& M. Miller (Eds), Crime and criminals, contemporary and classic readings in criminology. New York: Oxford University Press.

Silverio, E. M., \& García, H. M. (2007). Autopercepción de adaptación y tristeza en la adolescencia: Influencia de género. Anales de Psicología, 23, 41-48.

Tong, L. S. J., \& Farrington, D. P. (2006). How effective is the "Reasoning and Rehabilitation" programme in reducing reofendig? A meta-analysis of evaluations in fuor countries. Psychology, Crime \& Law, 12(1), 3-24.

Tong, L. S. J., \& Farrington, D. P. (2008). Efectiveness of reasoning and rehabilitation in reducing reoffending. Psicothema, 20(1), 2028.

Van Voorhis, P., Braswell, M., \& Lester, D. (2004). Correctional counseling and rehabilitation (5th ed.). Cincinnati, $\mathrm{OH}$ : Anderson.
Wettermann, A., Schlafke, D., \& Fegert, J. M. (2012). The modification of criminogenic factors on addicted offenders. The effectiveness of the Reasoning and Reahabilitation Program. International Journal of Law and Psychiatry, 35, 202-206.

World Health Organization. (2014). Global status report on violence prevention 2014. Recuperado de http://www.who.int/violence_injury_prevention/violence/status_report/2014/en/

Recibido el 9 de agosto de 2015 Revisado el 5 de octubre de 2015 Aceptado el 5 de noviembre de 2015 